\title{
RESINA COMPOSTA DIRETA NO RECONTORNO ESTÉTICO DE CANINOS EM CASO DE AGENESIA DE INCISIVOS LATERAIS: RELATO DE CASO
}

\section{DIRECT COMPOSITE RESIN IN THE ESTHETIC RECONTOURING OF CANINES IN CASE OF LATERAL INCISOR AGENESIS: CASE REPORT}

\author{
Petrusky Karyny dos Santos Silva ${ }^{1}$ (D), Maiarali Bento ${ }^{1}$ (D), Gabriela Queiroz de Melo Monteiro² (D), \\ Márcia de Almeida Durão ${ }^{1,2^{*}}$ (iD \\ ${ }^{1}$ Centro Universitário Mauricio de Nassau - Unidade Graças, Recife, PE, Brasil. \\ ${ }^{2}$ Universidade de Pernambuco, Recife, PE, Brasil. \\ *marciadurao.fop@gmail.com
}

\section{RESUMO}

A busca por um sorriso harmonioso tem sido mais frequente, o que leva os cirurgiões dentistas a se especializarem. Visando aprimorar suas habilidades e conhecimentos, dispondo assim de diversas técnicas para realizar tratamentos estéticos com excelência. A agenesia dentária é a ausência completa do dente em seu estágio embriológico. A etiologia mais aceita para explicar a ocorrência das anomalias dentárias é a alteração genética. Sendo esta uma das principais causas de insatisfação estética, quando acomete dentes anteriores. O objetivo desse relato de caso é demonstrar a aplicação de resina composta direta no recontorno estético de caninos em caso de agenesia de incisivos laterais. Após planejamento e aprovação pela paciente, foi realizado o clareamento dental caseiro por duas semanas. Após quinze dias, foi confeccionada a guia de silicone baseada no enceramento diagnóstico. $\mathrm{Na}$ sequência, foi realizado um pequeno desgaste da crista vestibular dos caninos e posterior recontorno com resina composta. Restabelecidos todos os parâmetros estéticos do sorriso, a paciente demonstrou total satisfação com o resultado. A restauração direta com resina composta quando bem planejada e executada corretamente, é uma excelente alternativa para reanatomização dental, resgatando a forma, função e harmonia estética do sorriso.

Palavras-chave: Agenesia. Estética dentária. Reanatomização dental.

\section{ABSTRACT}

The search for a harmonious smile has been more frequent, which leads dental surgeons to specialize aiming to improve their skills and knowledge, thus having several techniques to perform esthetic treatments with excellence. Dental agenesis is the complete absence of the tooth in its embryological stage. The most accepted etiology to explain the occurrence of dental anomalies is genetic alteration. This is one of the main causes of esthetic dissatisfaction, when it affects anterior teeth. The objective of this case report is to demonstrate the application of direct composite resin in the esthetic recontouring of canines in a case of agenesis of lateral incisors. After planning and approval by the patient, homemade tooth whitening was performed for two weeks. After fifteen days, a silicone guide based on diagnostic waxing was made. Subsequently, the buccal crest of the canines was slightly worn followed by recontouring with composite resin. After all the esthetic parameters of smile were restored, the patient showed total satisfaction with the result. Direct restoration with composite resin, when well-planned and correctly performed, is an excellent alternative for dental reshaping, recovering the shape, function and esthetic harmony of the smile.

Keywords: Agenesis. Aesthetic dentistry. Dental reshaping. 


\section{INTRODUÇÃO}

A agenesia dentária é a ausência completa do dente em seu estágio embriológico. Sendo a etiologia mais aceita relacionada à fatores genéticos (RIBEIRO et al., 2011; SOUZA et al., 2012). O incisivo lateral superior é o segundo dente mais frequentemente acometido, após os terceiros molares (SALGADO et al., 2012). Há alguns fatores associados a agenesia, como doenças infecciosas (rubéola), fatores ambientais (quimioterapia/drogas), que podem de alguma forma afetar o desenvolvimento dental (SINHORI et al., 2016). Essa alteração acarreta em várias anormalidades, como diastemas, dentes conóides e retenção de dentes decíduos (COELHO et al., 2012). Com relação a prevalência da agenesia, um estudo relatou que na população portuguesa o gênero feminino é mais acometido e que o terceiro molar é mais freqüente seguido do incisivo lateral (CARVALHO et al., 2011).

Buscando uma visão mais ampla e multidisciplinar, o paciente deve ser analisado de forma integral para o planejamento de sua reabilitação (SANTOS et al., 2018). Dentre as alternativas de tratamento, o fechamento do espaço pode exigir uma série de procedimentos, como clareamento dental, correção ortodôntica da posição dos dentes, análise gengival, contatos oclusais, contatos proximais, dentre outros (MIRANDA et al., 2016). Outra alternativa é a abertura e manutenção de espaço visando reabilitação com próteses ou implantes (RIBAS, 2014).

Para o caso em que se opta pela mesialização dos caninos para substituição dos incisivos laterais ausente, a ortodontia pode não ser tão eficaz no restabelecimento dos contatos proximais, sendo necessário que tal ajuste seja feito por reanatomização dos dentes em questão (BONFIM et al., 2012). O êxito da reanatomização através de restaurações diretas ou indiretas exige que o profissional tenha conhecimento dos princípios de estética (macro e microestética) e oclusão.

As resinas compostas são uma boa opção de tratamento conservador (HOEPPNER et al., 2003). E a correta aplicação da técnica de estratificação possibilita maior mimetização dental, através da reprodução dos efeitos de opalescência, opacidade e translucidez (STEFANI et al., 2015). A busca por um sorriso harmonioso tem sido um grande incentivo para a indústria odontológica e para desenvolvimento de técnicas restauradoras. A nanotecnologia trouxe a redução do tamanho das partículas de carga que contribuíram com a melhora das propriedades mecânicas, estéticas e durabilidade das restaurações (AZEVEDO et al., 2015). Nesse contexto o objetivo desse estudo foi demonstrar através de um relato de caso a técnica para reanatomização de caninos em incisivos laterais, com resina composta direta.

\section{RELATO DE CASO}

Paciente de 33 anos, gênero feminino, leucodérmica, procurou a Clínica Escola do Centro Universitário Mauricio de Nassau de Recife buscando um tratamento para harmonizar seu sorriso, uma vez que apresentava agenesia dos incisivos laterais superiores (Figura 1A), constatado através de exames radiográficos. A paciente relatou já ter feito tratamento ortodôntico por um curto período, visando fechamento dos diastemas nessa região. Tal tratamento normalmente deve conduzido de forma multidisciplinar visando atender as expectativas dos pacientes. Após aprovação do planejamento pela paciente foi realizado o registro inicial da cor dos caninos (A3), com a escala Vita (Figura 1B), na sequência, moldagem com alginato (Jeltrate, Dentsply, SP, Brasil), para confecção dos modelos de estudo.

Inicialmente foi realizado clareamento dental, com técnica caseira supervisionada, utilizando peróxido de carbamida 10\% (Power Bleaching, BM4, SC, Brasil), por duas semanas. Após 15 dias da finalização do clareamento, foi realizada a profilaxia com pedra pomes e escova de Robinson, também foi feita um novo registro da cor dos dentes, no qual os caninos apresentaram-se A1 na escala Vita. 
Foi realizado um leve desgaste na crista/aresta vestibular dos caninos com ponta diamantada 2200F e 3216F (KG Sorensen, Coitia, SP, Brasil), em alta rotação, sob refrigeração, visando planificar um pouco a superfície. A matriz de silicone foi adaptada aos dentes (Figura 2A), para verificar os espaços a serem preenchidos com a resina composta. Os pré-molares receberam apenas suave asperização com ponta diamantada 2200F (KG Soresen), com a finalidade de se obter um condicionamento eficaz sobre o esmalte, para melhor adesão.

Após instalação do isolamento absoluto modificado, feito o condicionamento com ácido fosfórico 37\% Condac (FGM, Joinville, SC, Brasil), por 30 segundos no esmalte dos caninos e prémolares (dentes 13, 14, 23 e 24). Após lavagem por 30 segundos e secagem vigorosa, foi aplicado com micropincel Cavibrush (FGM, Joinville, SC, Brasil), o sistema adesivo Prime e Bond 2.1 (Dentsply, Petrópolis, RJ, Brasil) seguindo o protocolo do fabricante. Aplicação por 20 segundos o adesivo abrangendo todas a superfície previamente condicionada dos caninos e pré-molares. Com um breve jato de ar (cerca de 5 segundos), foi feita volatização do solvente e remoção dos excessos do sistema adesivo, e posterior fotopolimerização por 10 segundos, com Emitter A (Fit-Schuster, Santa Maria, RS, Brasil), com potência de $800 \mathrm{~mW} / \mathrm{cm}^{2}$.

E em seguida, uma fina camada da resina composta para esmalte (Opallis, FGM, Joinville, SC, Brasil), cor A1, foi inserida na matriz de silicone,na área dos dentes 13, 14, 23 e 24 formando a região palatina e incisal, com espátulas Suprafil 2 (Golgran, São Caetano do Sul, SP, Brasil). A matriz foi adaptada aos referidos dentes e feita a fotopolimerização por 20 segundos. Na sequência, foram inseridos outros incrementos de resina composta de dentina A3 e A2 conferindo a opacidade e forma registrada na matriz de silicone. A escultura foi feita com espátulas supra fil e kit Esthetic Plus (TDV, Pomerode, SC, Brasil) e a textura (Figura 2A), foi conferida com pincel pêlo de marta $\mathrm{n}^{\circ} 4$ (Kota, Cotia, SP, Brasil). Cada incremento foi fotopolimerizado por 20 segundos até obter a cor e a forma planejada no modelo de estudo.

Em seguida foram removidos excessos de resina composta com lâmina de bisturi 12, e feita checagem da oclusão com papel carbono Contacto (Angelus, Londrina, PR-Brasil), com auxílio da pinça Muller. Foram analisados movimentos de oclusão, de protrusão (guia anterior) e de lateralidade, onde houve desoclusão apenas pelo contato dos caninos. Um pequeno desgaste foi feito apenas na região palatina dos caninos (13 e 23), com pontas diamantadas 3118FF, 2135FF (KG Soresen), em alta rotação, sob refrigeração. Deixando assim, a paciente numa oclusão confortável e adequada, mutuamente protegida, visando a longevidade e êxito do tratamento.

Após 24 horas, o acabamento mediato foi realizado em baixa rotação com pontas diamantadas 2135, 3195 e 3168 finas e extrafina (KG Sorensen). Também foram utilizados discos de lixa superfix do kit de acabamento e polimento (TDV, Pomerode, SC, Brasil). No polimento foram usadas pontas abrasivas de diferentes granulações e discos de feltro com pasta de polimento polimax do kit de acabamento e polimento (TDV, Pomerode, SC, Brasil) em baixa rotação.

A cor, a forma, a lisura e o brilho final das restaurações promoveram a naturalidade do sorriso, tão esperada pela paciente. Que demonstrou total satisfação, e ralatou ter um sonho realizado, com o sorriso harmonioso e natural (Figura 3), melhorando sua autoestima e o convívio social.

Figura 1 - Foto inicial (A) e registro inicial da cor (B)
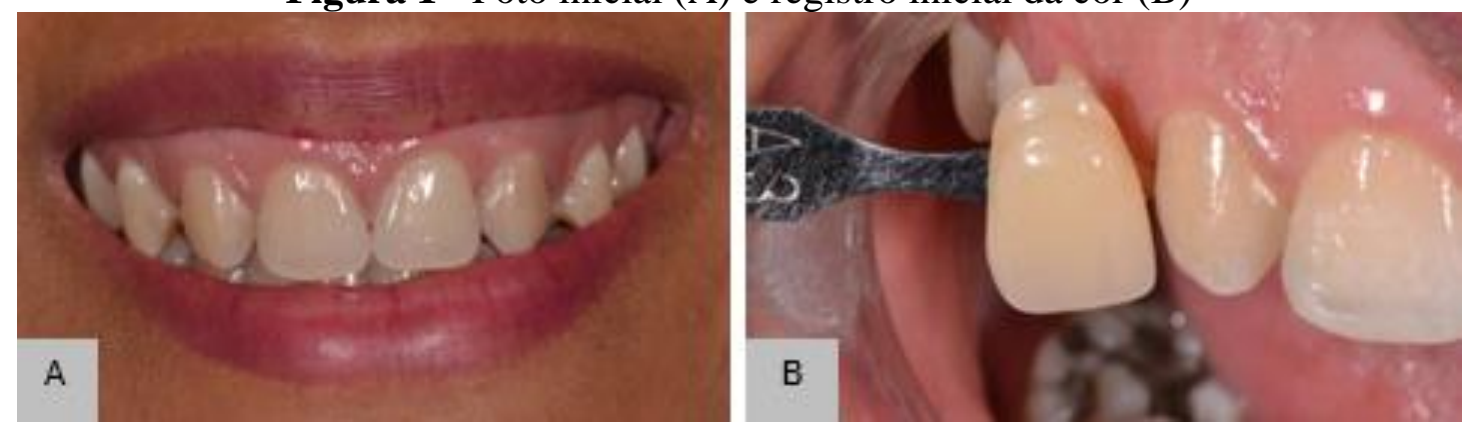

Fonte: os autores. 
Figura 2 - Guia de silicone adaptada (A) e Inserção da Resina composta (B)

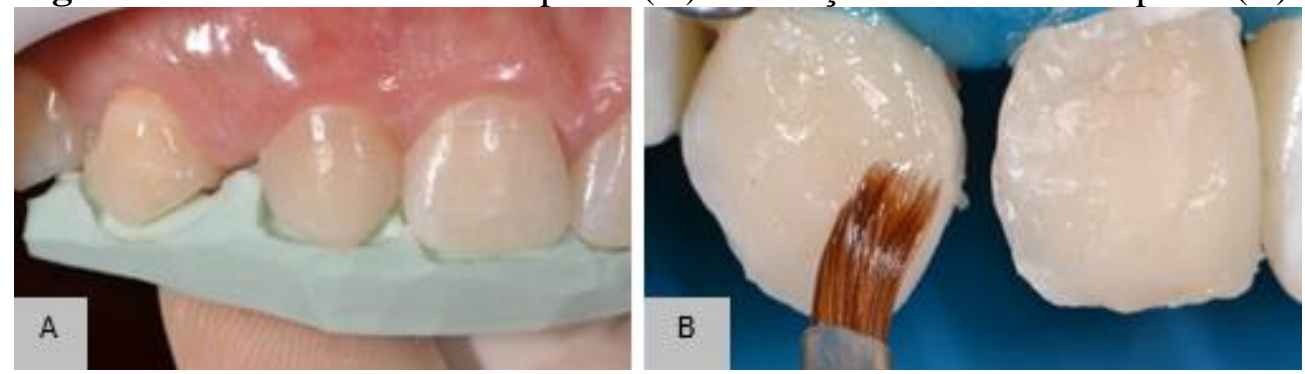

Fonte: os autores.

Figura 3 - Resultado final

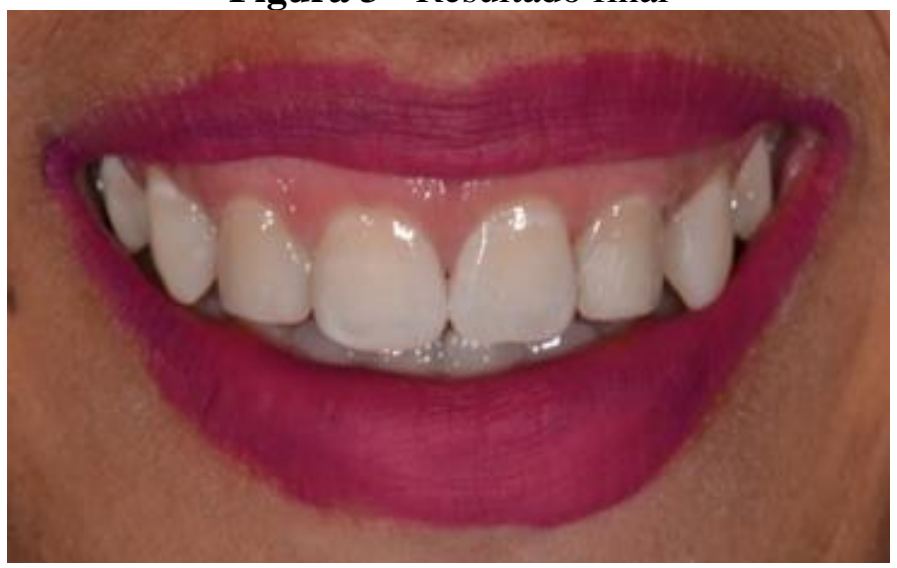

Fonte: os autores.

\section{DISCUSSÃO}

O tratamento da agenesia dental dispõe de algumas opções de tratamento como as restaurações diretas e as indiretas. O desenvolvimento dos compósitos restauradores aumentou e aprimorou algumas propriedades antes deficientes como a lisura, a estabilidade de cor e durabilidade das resinas (AZEVEDO et al., 2015). A nanotecnologia tem contribuído para esse progresso, o reduzido tamanho das partículas de carga promove maior resistência mecânica, maior durabilidade e excelente polimento (AZEVEDO et al., 2015). Portanto, as propriedades da resina composta selecionada são importantes para o sucesso e longevidade do tratamento (DAVISSON et al., 2016; GIMENEZ, 2016).

Dentre os critérios para escolha do material restaurador deste estudo foi considerado o tamanho das partículas de carga da resina composta (nanohíbridas), visto que, em geral, diferenciamse principalmente pelos componentes de carga inorgânica (BASEREN, 2004). As resinas compostas nanoparticuladas apresentam somente partículas esféricas de tamanho nanométrico, ao passo que as nanohíbridas contêm partículas irregulares microhíbridas além de nanopartículas. Ambas com propriedades satisfatórias para dentes anteriore e posteriors, além disso, utilizou-se as resinas compostas com cores e características ópticas de esmalte e de dentina.

A restauração direta em resina composta é uma opção viável para transformações cosméticas, tem como vantagem menor desgaste em estruturas dentais, menor tempo de procedimento, maior controle de cor e forma, um bom acabamento marginal e menor custo (RICCI et al., 2012). As reabilitações com técnicas indiretas também poderiam ser realizadas em casos de reanatomização. Porém algumas situações clínicas podem exigir preparo protético prévio, o que promoveria desgaste dental, considerando-as mais invasivas, mas isso não significa que sua aplicação esteja contraindicada (ATTIN et al., 2012; MESKO et al., 2016).

Embora não tenha sido necessário, neste caso clínico, a realização da recuperação da Dimensão vertical de Oclusão (DVO), é importante comentar toda análise realizada na oclusão da paciente. Entende-se por DVO, a posição vertical da mandíbula em relação à maxila, medida entre 
dois pontos definidos previamente, quando os dentes superiores e inferiores estão em contato intercuspídeos na posição de fechamento máximo. Segundo Pegoraro (2013), durante os movimentos mandibulares os dentes posteriores não podem participar da desoclusão, assim sendo, somente os dentes anteriores têm a função de desocluir. Os dentes que participam da guia anterior (GA), se portam como protetores para os dentes posteriores, durante os movimentos excêntricos, garantindo assim, o equilíbrio oclusal e muscular (PEGORARO, 2013). O movimento de lateralidade ocorre, no lado de trabalho, as cúspides com mesmo nome se relacionam, e dois tipos de guia de desoclusão são observadas (guia pelo canino e função em grupo). Já no lado de balanceio, as cúspides de nomes diferentes adotam uma relação de alinhamento. A guia ou desoclusão pelo canino, ocorre no movimento de lateralidade, quando o canino inferior desliza pela concavidade palatina do canino superior e os demais dentes não de contactam (CARDOSO, 2010). Neste caso clínico, as guias anterior, canino e lateralidade foram avaliadas e determinadas pelos movimentos excêntricos realizados pela própria paciente (HENRIQUES, 2003). A desoclusão foi mantida em canino (dentes 13 e 23), após realizada a reanatomização e posterior ajuste oclusal.

A estética pode ter sido a grande motivação da paciente em buscar tratamento, porém, todo planejamento foi feito baseado-se numa abordagem multidisciplinar. Afinal, o sucesso clínico e a longevidade são dependentes do controle da biomecânica da oclusão e domínio da técnica restauradora selecionada. Alguns dos argumentos a favor da indicação de restaurações diretas para reabilitação estética e funcional é possibilidade de reparo e a facilidade de substituição em caso de falhas mais extensas (GULAMALI et al., 2011). Em contrapartida, uma grande desvantagem das restaurações diretas com resina composta seria a dificuldade operatória, ou seja, o profissional precisa ter habilidade manual, ter um profundo conhecimento de oclusão e acerca das técinas adesivas restauradoras e assegurar um efetivo controle de umidade do campo operatório durante todo procedimento. Vale ressaltar, que todos esses requisitos, também se aplicam às restaurações indiretas.

Segundo Mesko et al. (2016), existem alguns profissionais receosos em realizar restaurações extensas com resinas compostas diretas e com certa desconfiança sobre o seu sucesso clínico. Esta técnica foi escolhida para a realização deste relato de caso, permitindo uma maior preservação da estrutura dental e restabelecimento estético-funcional de forma satisfatória.

\section{CONCLUSÃO}

Dessa forma, a restauração direta em resina composta, em casos de reanatomização dental, mostra-se uma excelente opção de tratamento. Uma vez que a técnica possibilita maior conservação da estrutura dental, promove cor e forma adequada, além de proporcionar lisura superficial e estética satisfatória.

\section{REFERÊNCIAS}

ATTIN, T. et al. Composite vertical bite reconstructions in eroded dentitions after 5.5 years: a case series. Journal of Oral Rehabilitation, v. 39, n. 1, p. 73-79, 2012.

AZEVEDO, N. et al. Otimização do sorriso com restaurações diretas de compósitos resinoso nanoparticulados. UNOPAR Científica. Ciências Biológicas e da Saúde, v. 17, n. 1, p. 43-49, 2015.

BASEREN, M. Surface roughness of nanofill and nanohybrid composite resin and ormocer-based tooth-colored restorative materials after several finishing and polishing procedures. Journal of Biomaterials Applications, v. 19, n. 2, p. 121-134, 2004.

BONFIM, A. C. Z. et al. Anodontia parcial e suas possibilidades ortodônticas. Revista Uningá, v. 34, n. 1, p. 109-128, 2012. 
CARDOSO, A. C. Oclusão para você e para mim. São Paulo: Ed. Santos, 2010. 233p.

CARVALHO, S.; MESQUITA, P.; AFONSO, A. Prevalência das anomalias de número numa população portuguesa. Estudo radiográfico. Revista Portuguesa de Estomatologia, Medicina Dentária e Cirurgia Maxilofacial, v. 52, n. 1, p. 7-12, 2011.

COELHO, L. G. C. et al. Reanatomização estética em pacientes com hipodontia,dente conoide e permanência de elemento decíduo. RPG - Revista da Pós Graduação, v. 17, n. 4, p. 204-208, 2012.

DAVISSON, A. P. et al. Reabilitação estética do sorriso por meio de procedimento restaurador direto com resina composta nanoparticulada: relato de caso. Revista Odontológica do Brasil Central, v. 25, n. 72, p. 54-58, 2016.

GIMENEZ, F. N. A estética do sorriso. 2016. 64f. Monografia (Trabalho de Conclusão de Curso) Universidade Estadual de Londrina, Londrina, 2016.

GULAMALI, A. B. et al. Survival analysis of composite Dahl restorations provided to manage localised anterior tooth wear (ten year follow-up). British Dental Journal, v. 211, n. 4, e9, 2011.

HENRIQUES, S. E. F. Reabilitação oral: filosofia, planejamento e oclusão. Cap. 10, p. 207-230. São Paulo: Ed. Santos, 2003.

HOEPPNER, M. G. et al. Tratamento estético de dente com alteração cromática: faceta direta com resina composta. Publicatio UEPG: Ciências e Biológicas da Saúde, v. 9, n. 3/4, p. 67-72, 2003.

MESKO, M. E. et al. Rehabilitation of severely worn teeth: a systematic review of clinical studies. Journal of Dentistry, v. 48, p. 9-15, 2016.

MIRANDA, R. R. et al. Tratamento odontológico integrado com ênfase em estética: relato de caso. Revista Odontológica do Brasil Central, v. 25, n. 74, p. 162-169, 2016.

PEGORARO, L. F. et al. Prótese Fixa: Bases para o Planejamento em Reabilitação Oral, 2. ed. 488p. São Paulo: Artes Médicas, 2013.

RIBAS, A. G. Agenesia Dentária: Revisão de Literatura. 2014. 51f. Monografia (Graduação em Odontologia) - Universidade Federal de Santa Catarina, Florianópolis, 2014.

RICCI, W. A. et al. Transformação cosmética de caninos em incisivos laterais: uma abordagem sistemática. Revista Dental Press Estética, v. 9, n. 3, p. 106-115, 2012.

RIBEIRO, L. N. S. et al. Aspectos clínicos e moleculares da agenesia dentária congênita. Revista de Odontologia da Universidade Cidade de São Paulo, v. 23, n. 2, p. 96-106, 2011.

SALGADO, H.; MESQUITA, P.; AFONSO, A. Agenesia do incisivo lateral superior- a propósito de um caso clínico. Revista Portuguesa Estomatologia, Medicina Dentária e Cirurgia Maxilofacial, v. 53, n. 3, p. 165-169, 2012.

SANTOS, M. R.; SILVA, M. M. Reabilitação protética em paciente portadora de agenesia dentária: relato de caso. Revista da Academia Brasileira de Odontologia, v. 27, n. 1, p. 36-41, 2018. 
SINHORI, B. S.; STOLF, S. C.; ANDRADA, M. A. C. Reanatomização estética de caninos em caso de agenesia de incisivos laterais. Clinica-Internacional Journal of Brazilian Dentistry, v. 12, n. 1, p. 58-64, 2016.

SOUZA, S. M. et al. Análise radiográfica de agenesia dentária. Archiev Oral Research, v. 8, n. 3, p. 197-203, 2012.

STEFANI, A. et al. Abordagem Multidisciplinar no Tratamento Estético Odontológico. Revista Associação Paulista de Cirurgiões Dentistas, v. 69, n. 1, p. 43-47, 2015. 